This item was submitted to Loughborough's Research Repository by the author.

Items in Figshare are protected by copyright, with all rights reserved, unless otherwise indicated.

\title{
A disturbance observer-based current-constrained controller for speed regulation of PMSM systems subject to unmatched disturbances
}

PLEASE CITE THE PUBLISHED VERSION

https://doi.org/10.1109/tie.2020.3005074

\section{PUBLISHER}

Institute of Electrical and Electronics Engineers (IEEE)

VERSION

AM (Accepted Manuscript)

\section{PUBLISHER STATEMENT}

(c) 2020 IEEE. Personal use of this material is permitted. Permission from IEEE must be obtained for all other uses, in any current or future media, including reprinting/republishing this material for advertising or promotional purposes, creating new collective works, for resale or redistribution to servers or lists, or reuse of any copyrighted component of this work in other works.

\section{LICENCE}

\section{All Rights Reserved}

\section{REPOSITORY RECORD}

Dai, Chen, Tianliang Guo, Jun Yang, and Shihua Li. 2020. "A Disturbance Observer-based Currentconstrained Controller for Speed Regulation of PMSM Systems Subject to Unmatched Disturbances". Loughborough University. https://hdl.handle.net/2134/16811338.v1. 


\title{
A Disturbance Observer-Based Current-Constrained Controller for Speed Regulation of PMSM Systems Subject to Unmatched Disturbances
}

\author{
Chen Dai, Member, IEEE, Tianliang Guo, Jun Yang, Senior Member, IEEE, and Shihua Li, Fellow, IEEE
}

\begin{abstract}
The speed regulation problem of permanent magnet synchronous motor (PMSM) system is investigated under a non-cascade structure. Despite its superiority in straightforward control design, simple parameter adjustment and satisfying system dynamic performance, the structure brings two problems: the overcurrent protection and unmatched disturbance rejection. Under this structure, the current cannot any more be restricted by a reference value, and ought to be constrained within a certain range to ensure the circuit safety. Besides, unmatched disturbances, mainly caused by external load torques, may result in undesired interference and violate the constraint requirement since they affect the current directly via the same channel. Toward that end, a novel current-constrained control algorithm is designed to tackle the current constraint and unmatched disturbances simultaneously. A disturbance observer (DOB) is utilized for unmatched disturbance estimation. A constraint coping mechanism is constructed to restrict the current. Then, a key nonlinear item is proposed by augmenting the disturbance estimation and the constrained current. Finally, a composite controller is proposed with concise structure and rigorous closed-loop stability analysis. Numerical and experimental tests further validate that the proposed control approach achieves promising speed tracking performance and guarantees the current constraint in the presence of unmatched disturbances.
\end{abstract}

Index Terms-Permanent magnet synchronous motor, speed regulation, current constraint, unmatched disturbance, disturbance observer.

\section{INTRODUCTION}

$\mathbf{P}$ ERMANENT magnet synchronous motors (PMSMs) have been paid abundant attention and broadly adopted in various practical applications, such as power generations, robotics and electric vehicles [1]-[3], because of their compact structure, high efficiency and power density [4].

With technical development, there is little difference between the control periods of the inner and outer-loops. As an

The work was supported in part by the National Natural Science Foundation (NNSF) of China under Grant 61973080 and Grant 61973081 (Corresponding author: Shihua Li).

C. Dai, T. Guo, J. Yang and S. Li are with School of Automation, Southeast University, Key Laboratory of Measurement and Control of CSE, Ministry of Education, Nanjing 210096, P.R. China (e-mail: Ish@seu.edu.cn). alternative, the non-cascade structure is applied with straightforward control design, simple parameter adjustment and satisfying system dynamic performance [5]-[9].

Despite its advantages, the non-cascade structure inevitably brings about some problems:

1) The overcurrent protection: unlike the cascade structure, the current cannot be restricted by a reference value any more. In such cases, the current is considered as a system state. Thus, the overcurrent protection is not negligible, since it would cause performance degradation or even system damage [10];

2) The unmatched disturbance rejection: under the noncascade structure, disturbances in the current loop are unmatched, which enter the system via a different channel from the control input. Mainly caused by external load torques, unmodelled dynamics and parameter perturbations, the unmatched disturbances have negative impact on system performance and stability [11]. It is of significance but challenge to reject them, since they influence the current directly via the same channel and thus may violate the constraint. And it is hard to eliminate their influence from the output [12].

Traditional control approaches (e.g. PID) do not explicitly consider the above problems in the control design. Commonly, they restrict the current by selecting relatively conservative control parameters and reject the disturbances in a robust way. Consequently, they cannot balance well among requirements of system dynamic performance, satisfaction of the current constraint and unmatched disturbance rejection ability [13].

Nowadays, various elegant approaches have been investigated. One idea is to seek the invariant set (IS) in the domain of constraints [14], [15]. The states starting from the IS will always remain in it. Since the IS estimation depends highly on the choice of Lyapunov functions, it is challenging and requires considerable calculation to find the maximal positive IS [16]. Another effective way is the model predictive control (MPC), which transforms control design issues into optimization problems [5], [6], [17]-[19]. By constructing a cost function including the constrained current, the current is restricted by control actions that minimize the given cost function. This kind of constraints can be regarded as soft ones as the constraints are realized via an indirect manner [13], [20]. Besides, the barrier Lyapunov function (BLF) is widely exerted, which is related to the constrained state and will increase to infinity when the constraint is approached [21]- 
[23]. The BLF based backstepping control has had fruitful practical investigations, such as electrostatic microactuators [24], electro-hydraulic systems [25] and flexible crane systems [26]. Unfortunately, it is conservative to choose control parameters. Since in each recursive step, the virtual controller must be restricted in the constrained domain [23]. Moreover, few works have considered the control solutions to address unmatched disturbances, which have direct impact on the constrained state via the same channel.

Recently, the penalty term based state-constrained control has been researched, where a nonlinear function of the constrained state is used to regulate the control gain automatically [27], [28]. A current-constrained PID (CCPID) controller is implemented in PMSMs to restrict the current [28]. But the disturbance rejection issue is not explicitly taken into account in the control design. The unmatched disturbance is simply counteracted by an integral action. This may bring negative effect on control performance and parameter tuning due to performance couplings caused by integral actions [13].

To improve the disturbance rejection ability, several control strategies have combined the disturbance observer (DOB) technology [29]-[35] with the penalty term [36]-[38]. However, the above methods do not tackle the unmatched disturbance issue in essence. To be specific, in [36], only the matched disturbance is considered and handled in currentconstrained DC-DC converter systems. In [37], [38], unmatched disturbances in PMSMs are considered within the current-constrained control strategies, but lack of rigorous closed-loop stability and performance analysis. To conclude, for many motion control systems with second-order dynamics, the overcurrent protection together with unmatched disturbance problems are neither explicitly considered nor fundamentally solved. Furthermore, it is significant and meaningful to investigate a general control algorithm for state-constrained nonlinear systems subject to unmatched disturbances.

This paper simultaneously tackles the overcurrent protection and unmatched disturbance rejection problems for speed regulation of PMSMs. To begin with, a DOB is designed to estimate the unmatched disturbance. Following the observer design, a key nonlinear item including a feed-forward compensation part and a constraint coping mechanism part, is proposed by augmenting the disturbance estimation and the constrained state. The former is introduced to attenuate unmatched disturbances, which is related to the system structure. The latter is utilized to restrict the current, which is with respect to the current and automatically increases when the current tends to its boundary. As such, a novel DOB-based current-constrained controller (DOB-CC) is obtained. Robust closed-loop stability analysis, together with numerical and experimental tests have verified its effectiveness. The main contributions and novelties of the paper are as follows:

1) A general control algorithm is investigated for stateconstrained nonlinear systems subject to unmatched disturbances, which affect the constrained state directly via the same channel. To the best of our knowledge, it is the first time in the literature to explicitly solve this problem with rigorous closed-loop stability analysis;
2) For speed regulation of PMSMs, a new current-constrained control algorithm is proposed to simultaneously fulfill the overcurrent protection task and compensate undesirable effects of unmatched load torque variations;

3) By virtue of a key nonlinear item via a DOB and a constraint coping mechanism, the proposed control algorithm not only exhibits promising control performance, but also guarantees the current constraint effectively, even in the presence of unmatched disturbances;

4) The proposed control algorithm can be easily extended to many motion control systems with second-order dynamics due to the concise control structure and the complete theoretic guarantees.

\section{Problem Formulation}

\section{A. Mathematical Model}

The mathematical model of a surface-mounted PMSM in the $\mathrm{d}-\mathrm{q}$ frame is formulated as [11], [28]

$$
\left\{\begin{array}{l}
\dot{\omega}=\frac{1}{J}\left(n_{p} \psi_{f} i_{q}-B \omega-T_{L}\right), \\
\dot{i_{d}}=\frac{1}{L}\left(-R_{s} i_{d}+n_{p} \omega L i_{q}+u_{d}\right), \\
\dot{i_{q}}=\frac{1}{L}\left(-R_{s} i_{q}-n_{p} \omega L i_{d}-n_{p} \psi_{f} \omega+u_{q}\right),
\end{array}\right.
$$

where $u_{d}, u_{q}, i_{d}$ and $i_{q}$ are the stator voltages and currents of the $d$ and $q$-axes, respectively; $\omega$ is the angular velocity; $n_{p}$ is the number of pole pairs; $R_{s}$ is the stator resistance; $L$ is the stator inductance; $\psi_{f}$ is the rotor flux linkage; $T_{L}$ is the load torque; $J$ is the rotor inertia and $B$ is the viscous frictional coefficient.

Here, the reference value of the d-axis current $i_{d}^{*}$ is set to zero to obtain the maximum torque-to-current ratio [39]. If the controller of $i_{d}$ loop works well, one obtains $i_{d} \doteq i_{d}^{*}=0$. In such case, system (1) reduces to

$$
\left\{\begin{array}{l}
\dot{\omega}=\frac{1}{J}\left(n_{p} \psi_{f} i_{q}-B \omega-T_{L}\right), \\
\dot{i_{q}}=\frac{1}{L}\left(-R_{s} i_{q}-n_{p} \psi_{f} \omega+u_{q}\right) .
\end{array}\right.
$$

Suppose that the current $i_{q}$ is bounded by a constant $c>0$, which is a safety threshold and often selected as $2 \sim 3$ times of the rated current in industrial applications [10]. Since the load torque variations influence the current directly, a large load torque change may violate the current constraint. Thus, it is reasonable to assume that the load torque $T_{L}$ changes within a range related to $c$ to satisfy the constraint requirements.

Assumption 1: The load torque $T_{L}$ has a positive boundary, which is defined as $\bar{D}=\left(n_{p} \psi_{f} c-B \omega_{\text {ref }}\right) / 2$.

Remark 1: Since the rated electromagnetic torque $n_{p} \psi_{f} I_{N}$ is obviously larger than the maximum viscous torque $B \omega_{\text {ref }}$, where $I_{N}$ is the rated current. With the selection of $c$, i.e., $c>I_{N}$, it yields that $n_{p} \psi_{f} c>B \omega_{r e f}$, thus $\bar{D}>0$.

\section{B. Control-Oriented Model}

The control objective is to make the rotating speed track a given reference signal, ensuring the current constraint in the presence of unmatched disturbances.

Firstly, the rotating speed tracking error is defined as $e_{1}=$ $\omega_{\text {ref }}-\omega$, where $\omega_{\text {ref }}$ is the constant reference rotating speed. 
Then, defining $e_{2}=-n_{p} \psi_{f} i_{q}+B \omega_{r e f}$ as the system state, and the control-oriented model is written as

$$
\left\{\begin{array}{l}
\dot{e}_{1}=\frac{1}{J}\left(-a_{1} e_{1}+e_{2}+d\right), \\
\dot{e}_{2}=\frac{1}{L}\left[f\left(e_{1}, e_{2}\right)+u\right] \\
y=e_{1}
\end{array}\right.
$$

where $u=b u_{q}$ is the control input, and $y$ is the system output, with

$$
\begin{aligned}
& a_{1}=B, b=-n_{p} \psi_{f}, d=T_{L}, \\
& f\left(e_{1}, e_{2}\right)=-n_{p}^{2} \psi_{f}^{2} e_{1}-R_{s} e_{2}+\left(n_{p}^{2} \psi_{f}^{2}+R_{s} B\right) \omega_{r e f} .
\end{aligned}
$$

For simplicity of the theoretical analysis, denoting $M_{1}=$ $n_{p} \psi_{f} c+B \omega_{r e f}$ and $M_{2}=n_{p} \psi_{f} c-B \omega_{r e f}$. From the previous analysis, we have $M_{1}>0$ and $M_{2}>0$. In this way, the current constraint $\left|i_{q}\right|<c$ is equivalent to the state constraint $e_{2} \in\left(-M_{2}, M_{1}\right)$. And the control problem is transformed to stabilize $e_{1}$ in the presence of the state constraint $e_{2} \in$ $\left(-M_{2}, M_{1}\right)$ and the unmatched disturbance $d$.

\section{Motivation of Control Design}

To explain our motivation more illustratively, two kinds of the existing control methods are analyzed theoretically.

1) The Current-Constrained PID (CCPID) Controller:

The CCPID controller is expressed as [28]

$$
\begin{aligned}
u= & -k_{P} e_{1}-k_{I} \int_{0}^{t} e_{1} \mathrm{~d} \tau-f\left(e_{1}, e_{2}\right) \\
& -\left[k_{D}+\frac{l}{\left(M_{1}-e_{2}\right)\left(M_{2}+e_{2}\right)}\right] e_{2} \\
= & -k_{P} e_{1}-k_{I} \int_{0}^{t} e_{1} \mathrm{~d} \tau-k_{D}^{\prime} e_{2}-f\left(e_{1}, e_{2}\right),
\end{aligned}
$$

where $k_{D}^{\prime}=k_{D}+\frac{l}{\left(M_{1}-e_{2}\right)\left(M_{2}+e_{2}\right)}$, and $k_{P}, k_{D}, k_{I}$, $l$ are positive control parameters. Since the penalty term $\frac{l}{\left(M_{1}-e_{2}\right)\left(M_{2}+e_{2}\right)}$ increases to infinity when the current tends to its boundary, the control action can restrict the current.

Combining (3) and (5), it yields

$$
\dot{e}_{2}=\frac{1}{L}\left(-k_{P} e_{1}-k_{I} \int_{0}^{t} e_{1} \mathrm{~d} \tau-k_{D}^{\prime} e_{2}\right),
$$

and it can be further obtained that

$$
\dddot{e}_{1}+a_{2} \ddot{e}_{1}+a_{3} \dot{e}_{1}+a_{4} e_{1}=\frac{k_{D}}{L J} \dot{d}+\frac{1}{J} \ddot{d},
$$

where $a_{2}=\frac{a_{1}}{J}+\frac{k_{D}^{\prime}}{L}, a_{3}=\frac{k_{P}}{L J}+\frac{a_{1} k_{D}^{\prime}}{L J}$, and $a_{4}=\frac{k_{I}}{L J}$.

Remark 2: Although the current can be restricted, the controller can hardly react promptly to alleviate the influence of $\dot{d}$ and $\ddot{d}$ on the tracking error $e_{1}$. Moreover, the integral action may bring adverse effects, e.g., large overshoots and long settling time.

2) The Traditional DOBC:

The controller is expressed as [31]

$$
u=-k_{1} e_{1}-k_{2}\left(e_{2}+\hat{d}\right)-f\left(e_{1}, e_{2}\right),
$$

where $k_{1}, k_{2}$ are positive control parameters, and $\hat{d}$ is the disturbance estimation provided by a DOB.
Combining (3) and (8), it yields

$$
\left\{\begin{array}{l}
\dot{e}_{1}=\frac{1}{J}\left(-a_{1} e_{1}+e_{2}+d\right) \\
\dot{e}_{2}=\frac{1}{L}\left[-k_{1} e_{1}-k_{2}\left(e_{2}+\hat{d}\right)\right] .
\end{array}\right.
$$

Denoting $e_{d}=d-\hat{d}$ as the estimation error, one obtains

$$
\ddot{e}_{1}+k_{1}^{\prime} \dot{e}_{1}+k_{2}^{\prime} e_{1}=\frac{1}{J} \dot{d}+\frac{k_{2}}{L J} e_{d},
$$

where $k_{1}^{\prime}=\frac{a_{1}}{J}+\frac{k_{2}}{L}$ and $k_{2}^{\prime}=\frac{k_{1}}{L J}+\frac{a_{1} k_{2}}{L J}$.

It is supposed that $d$ has a constant steady-state value and is accurately reconstructed, then it yields

$$
\ddot{e}_{1}+k_{1}^{\prime} \dot{e}_{1}+k_{2}^{\prime} e_{1}=0
$$

which implies that $e_{1}$ can converge to zero asymptotically by choosing the control parameters properly.

Given that $e_{1}=0$, we have

$$
\dot{e}_{2}=-\frac{k_{2}}{L}\left(e_{2}+\hat{d}\right) \text {. }
$$

Remark 3: It implies that the DOBC cannot rigidly ensure the current constraint. Usually, it selects relatively conservative control parameters to avoid excessive current. But this inevitably sacrifices the dynamic performance to some extent.

\section{Controller Design}

In this section, a DOB is firstly utilized for the unmatched disturbance estimation. Secondly, a key nonlinear item containing a feed-forward compensation part and a constraint coping mechanism part is proposed. Then, a composite controller is developed with rigorous closed-loop stability analysis.

\section{A. Design of the $D O B$}

To acquire the estimation value $\hat{d}$ of the unmatched disturbance $d$ in system (3), the DOB is designed as [31]

$$
\left\{\begin{array}{l}
\dot{z}=-\frac{p}{J} z+\frac{p}{J}\left(a_{1} e_{1}-e_{2}-p e_{1}\right) \\
\hat{d}=z+p e_{1}
\end{array}\right.
$$

where $z$ is the observer state, and $p>0$ is the observer gain, respectively.

Assumption 2: The derivative of $d$ in system (3) has a positive boundary, which is defined as $d_{d}^{*}=\sup _{t>0}|\dot{d}(t)|$.

Lemma 1 [29]: For system (3) under Assumptions 1 and 2 , if $d$ satisfies that $\lim _{t \rightarrow \infty} \dot{d}(t)=0$, then the estimation error $e_{d}=d-\hat{d}$ converges to zero asymptotically.

According to (13), one obtains

$$
\dot{e}_{d}=\dot{d}-\dot{\hat{d}}=\dot{d}-\frac{p}{J} e_{d} .
$$

Under Assumption 2, it yields

$$
\left|e_{d}(t)\right| \leq e^{-\frac{p}{J} t} e_{d}(0)+\frac{J}{p}\left(1-e^{-\frac{p}{J} t}\right) d_{d}^{*} \leq D,
$$

where $D=\sup _{t>0}\left|e_{d}(t)\right|$ is the upper bound of $e_{d}(t)$ and it decreases as $p$ is increasing. 


\section{B. Design of the Composite Controller}

For system (3), the DOB-CC controller is designed as

$$
\begin{aligned}
u= & -k_{1} e_{1}-f\left(e_{1}, e_{2}\right) \\
& -\left[k_{2}+\frac{l}{\left(M_{1}-e_{2}\right)\left(M_{2}+e_{2}\right)}\right]\left(e_{2}+\hat{d}\right),
\end{aligned}
$$

and the actual control law is expressed as

$$
\begin{aligned}
u_{q}= & \frac{k_{1}}{n_{p} \psi_{f}}\left(\omega_{r e f}-\omega\right)+R_{s} i_{q}+n_{p} \psi_{f} \omega \\
& +\frac{1}{n_{p} \psi_{f}}\left(k_{2}+\frac{l}{c^{2}-i_{q}^{2}}\right)\left(B \omega_{r e f}-n_{p} \psi_{f} i_{q}+\hat{d}\right),
\end{aligned}
$$

where $k_{1}, k_{2}$, and $l$ are positive control parameters.

In (16), the constraint coping mechanism $\frac{l}{\left(M_{1}-e_{2}\right)\left(M_{2}+e_{2}\right)}$ is a nonlinear continuous function when $e_{2} \in\left(-M_{2}, M_{1}\right)$. If $i_{q}$ tends to the limitation $\pm c$, i.e. the state $e_{2}$ tends to the barrier boundary $M_{1}$ or $-M_{2}$, the function will tend to infinity. In this manner, the controller restricts the current effectively by regulating the controller gain automatically.

Combining (14) and (16) with (3), the closed-loop system is written as

$$
\left\{\begin{array}{l}
\dot{e}_{1}=-\frac{a_{1}}{J} e_{1}+\frac{1}{J}\left(e_{2}+\hat{d}\right)+\frac{1}{J} e_{d}, \\
\dot{e}_{2}=-\frac{k_{1}}{L} e_{1}-\frac{1}{L}\left[k_{2}+\frac{l}{\left(M_{1}-e_{2}\right)\left(M_{2}+e_{2}\right)}\right]\left(e_{2}+\hat{d}\right), \\
\dot{e}_{d}=\dot{d}-\frac{p}{J} e_{d},
\end{array}\right.
$$

where $e=\left[e_{1}, e_{2}, e_{d}\right]^{T}$ is denoted as the state vector.

Theorem 1: Under Assumptions 1 and 2, if the initial state $e_{2}(0) \in\left(-M_{2}, M_{1}\right)$, i.e., $i_{q}(0) \in(-c, c)$, the state $e$ of the closed-loop system (18) asymptotically converges to a bounded compact set $\Omega_{B}=\left\{e \mid \alpha V(e) \leq \frac{1}{2} d_{d}^{* 2}\right\}$, where $\alpha>$ 0 is specified later. Meanwhile, the condition $e_{2} \in\left(-M_{2}, M_{1}\right)$ holds. Namely, the rotating speed $\omega$ asymptotically tracks the reference value $\omega_{\text {ref }}$ to a bounded compact set, and the current constraint $\left|i_{q}\right|<c$ is guaranteed.

Proof: Defining $T$ as a positive constant or $T=+\infty$. It is assumed that the condition $e_{2}(t) \in\left(-M_{2}, M_{1}\right)$ is satisfied during $t \in[0, T)$. There are three steps in the proof.

Step 1: to prove that the states of system (18) are bounded, when $t \in[0, T)$.

The candidate Lyapunov function is chosen as

$$
V(e)=\frac{k_{1} J}{2 L} e_{1}^{2}+\frac{1}{2}\left(e_{2}+\hat{d}\right)^{2}+\frac{1}{2} e_{d}^{2} .
$$

Taking the derivative of $V(e)$ along system (18), one obtains

$$
\begin{aligned}
\dot{V}(e)= & \frac{k_{1} J}{L} e_{1} \dot{e}_{1}+\left(e_{2}+\hat{d}\right)\left(\dot{e}_{2}+\dot{\hat{d}}\right)+e_{d} \dot{e}_{d} \\
= & -\frac{a_{1} k_{1}}{L} e_{1}^{2}+\frac{k_{1}}{L} e_{1} e_{d}-\frac{k_{2}}{L}\left(e_{2}+\hat{d}\right)^{2}+\frac{p e_{d}}{J}\left(e_{2}+\hat{d}\right) \\
& -\frac{p}{J} e_{d}^{2}+e_{d} \dot{d}-\frac{l\left(e_{2}+\hat{d}\right)^{2}}{L\left(M_{1}-e_{2}\right)\left(M_{2}+e_{2}\right)} \\
\leq & -\frac{1}{L}\left(a_{1} k_{1}-\frac{1}{2}\right) e_{1}^{2}-\left(\frac{k_{2}}{L}-\frac{p}{2 J}\right)\left(e_{2}+\hat{d}\right)^{2} \\
& -\frac{1}{2}\left(\frac{p}{J}-\frac{k_{1}^{2}}{L}-1\right) e_{d}^{2}+\frac{1}{2} d_{d}^{* 2} .
\end{aligned}
$$

If the control parameters are selected such that $\frac{1}{2 a_{1}}<k_{1}<$ $\sqrt{\left(\frac{p}{J}-1\right) L}$ and $k_{2}>\frac{p L}{2 J}$, then we have

$$
\dot{V}(e) \leq-\alpha V(e)+\frac{1}{2} d_{d}^{* 2},
$$

where $\alpha=\min \left\{\frac{2 a_{1}}{J}-\frac{1}{J k_{1}}, \frac{2 k_{2}}{L}-\frac{p}{J}, \frac{p}{J}-\frac{k_{1}^{2}}{L}-1\right\}$ is positive. According to the Lyapunov theory, one can conclude that the states of system (18) are bounded, when $t \in[0, T)$. Furthermore, we suppose that $e_{1}$ is bounded by a positive constant $A_{0}=\sup _{t>0}\left|e_{1}(t)\right|, t \in[0, T)$.

Step 2: to prove that the condition $e_{2} \in\left(-M_{2}, M_{1}\right)$ holds, i.e., the current constraint is ensured all the time;

For definition clarity, denoting $G=(-\infty,+\infty) \times$ $\left(-M_{2}, M_{1}\right) \times[-D, D]$ as the corresponding domain to the feasible zone of system (2) in Fig. 1(a). In Fig. 1(b), $G_{1}$ (blue, top) and $G_{2}$ (green, bottom) represent the corresponding values of $e$ when $i_{q}$ is close to its boundaries $\pm c$, respectively; and $G_{3}$ (white, middle) is the rest part satisfying $\left|i_{q}\right|<c$, which will be specified later.

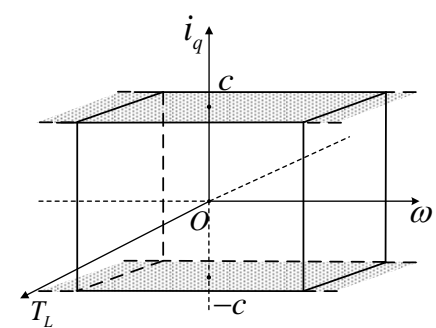

(a)

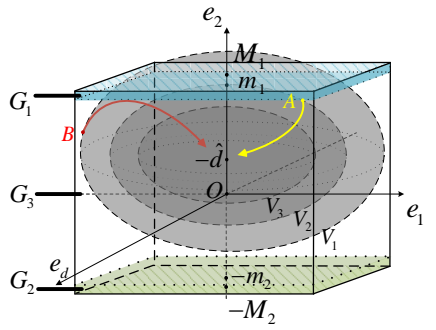

(b)
Fig. 1. System state graphs: (a) the feasible zone of the PMSM system (2), (b) states of the closed-loop system (18).

Based on Step 1, denoting $G_{1}=\left[-A_{0}, A_{0}\right] \times\left[m_{1}, M_{1}\right) \times$ $[-D, D], G_{2}=\left[-A_{0}, A_{0}\right] \times\left(-M_{2},-m_{2}\right] \times[-D, D]$, and $G_{3}=\left[-A_{0}, A_{0}\right] \times\left(-m_{2}, m_{1}\right) \times[-D, D]$, where $m_{1}$ and $m_{2}$ are chosen as

$$
\begin{aligned}
& m_{1}=\max \left\{\left|e_{2}(0)\right|, N_{1}, \tilde{D}\right\}>0, \\
& m_{2}=\max \left\{\left|e_{2}(0)\right|, N_{2}, \tilde{D}\right\}>0,
\end{aligned}
$$

with

$$
\begin{aligned}
& N_{1}=\frac{M_{1} \eta+\tilde{D} l}{\eta+l}, N_{2}=\frac{M_{2} \eta+\tilde{D} l}{\eta+l}, \\
& \eta=k_{1} A_{0}\left(M_{1}+M_{2}\right), \tilde{D}=D+\bar{D} .
\end{aligned}
$$

To prove that the condition $e_{2}(t) \in\left(-M_{2}, M_{1}\right)$ holds when $t \in[0, T)$, the following three cases are discussed to prove that the states starting from $G_{1}$ and $G_{2}$ cannot cross the restricted boundaries when $t \in[0, T)$.

- Case 1: If $e(t) \in G_{1}$, where $e_{2}(t) \in\left[m_{1}, M_{1}\right), t \in[0, T)$. Taking the derivative of $e_{2}(t)$, it yields

$$
\begin{aligned}
\dot{e}_{2} & =-\frac{k_{1} e_{1}}{L}-\frac{e_{2}+\hat{d}}{L}\left[k_{2}+\frac{l}{\left(M_{1}-e_{2}\right)\left(M_{2}+e_{2}\right)}\right] \\
& \leq \frac{k_{1} A_{0}}{L}-\frac{l\left(e_{2}-\tilde{D}\right)}{L\left(M_{1}+M_{2}\right)\left(M_{1}-e_{2}\right)}<0,
\end{aligned}
$$


and we have $e_{2}(t) \dot{e}_{2}(t)<0, t \in[0, T)$. It implies that the condition $e_{2}(t)<M_{1}, t \in[0, T)$ cannot be violated and the state $e$ will enter $G_{3}$ according to Step 1. In Fig. 1(b), the trajectory of Point A is drawn for instance.

- Case 2: If $e(t) \in G_{2}$, where $e_{2}(t) \in\left(-M_{2},-m_{2}\right], t \in$ $[0, T)$. Taking the derivative of $e_{2}(t)$, it yields

$$
\begin{aligned}
\dot{e}_{2} & =-\frac{k_{1} e_{1}}{L}-\frac{e_{2}+\hat{d}}{L}\left[k_{2}+\frac{l}{\left(M_{1}-e_{2}\right)\left(M_{2}+e_{2}\right)}\right] \\
& \geq-\frac{k_{1} A_{0}}{L}-\frac{l\left(e_{2}+\tilde{D}\right)}{L\left(M_{1}+M_{2}\right)\left(M_{2}+e_{2}\right)}>0,
\end{aligned}
$$

and we have $e_{2}(t) \dot{e}_{2}(t)<0, t \in[0, T)$. One obtains that the condition $e_{2}(t)>-M_{2}, t \in[0, T)$ cannot be violated and the state $e$ will enter $G_{3}$ likewise.

- Case 3: If $e(t) \in G_{3}$, where $e_{2}(t) \in\left(-m_{2}, m_{1}\right)$, $t \in[0, T)$, then the condition $e_{2}(t) \in\left(-M_{2}, M_{1}\right)$ is originally satisfied. Considering Cases 1 and 2 , if $e$ enters $G_{1}$ or $G_{2}$, it will finally move into $G_{3}$. And (21) reveals that infinite switches between Case 3 and Case 1/Case 2 cannot happen. In Fig. 1(b), the trajectory of Point B is drawn for instance.

Next, we will prove that $T=+\infty$, which indicates the satisfaction of the condition $e_{2} \in\left(-M_{2}, M_{1}\right)$ all the time.

It is assumed that for any $e(0) \in G, e(t)$ is the solution of system (18) on $G_{3}^{\prime}=\left(-A_{0}-1, A_{0}+1\right) \times\left(-M_{2}, M_{1}\right) \times$ $[-D, D] \subset G, t \in[0, T)$. Then, we have $e(t) \in G_{3} \subset G_{3}^{\prime}$, $t \in[0, T)$. Since it is defined that $T>0$ or $T=+\infty$, on the basis of the continuation theorem of solution, it is obtained that $T=+\infty$, Thus, $e_{2} \in\left(-M_{2}, M_{1}\right)$ always holds, i.e., the current constraint $\left|i_{q}\right|<c$ is satisfied all the time.

Step 3: to prove that the state e of system (18) converges to a bounded compact set asymptotically.

From the above analysis, it is obtained that (21) holds when $t \in[0,+\infty)$. Then, denoting a bounded compact set as $\Omega_{B}=$ $\left\{e \mid \alpha V(e) \leq \frac{1}{2} d_{d}^{* 2}\right\}$, one obtains that the state $e$ of system (18) converges to $\Omega_{B}$ asymptotically. As such, the rotating speed $\omega$ tracks the reference signal $\omega_{\text {ref }}$ to a bounded compact set asymptotically. This completes the proof.

Corollary 1: Under Assumptions 1 and 2, if the condition $\lim _{t \rightarrow \infty} \dot{d}(t)=0$ holds, and the initial state $e_{2}(0) \in\left(-M_{2}, M_{1}\right)$, i.e., $i_{q}(0) \in(-c, c)$, then the origin of the closed-loop system (18) is asymptotically stable and the condition $e_{2} \in$ $\left(-M_{2}, M_{1}\right)$ always holds. Namely, the rotating speed $\omega$ tracks the reference value $\omega_{\text {ref }}$ asymptotically, while the current constraint $\left|i_{q}\right|<c$ is guaranteed all the time.

Proof: The proof is analogous to that of Theorem 1, and thus is omitted here for space.

The control block diagram is presented in Fig. 2.

Remark 4: The proposed controller mainly focuses on handling the current constraint and unmatched disturbances for the speed regulation of PMSMs. When further considering the control input saturation, a safe distance away from the constraint boundary should be determined for the current, which helps provide a more flexible current constraint range for practical applications. Theoretical analysis will be investigated in the future work.

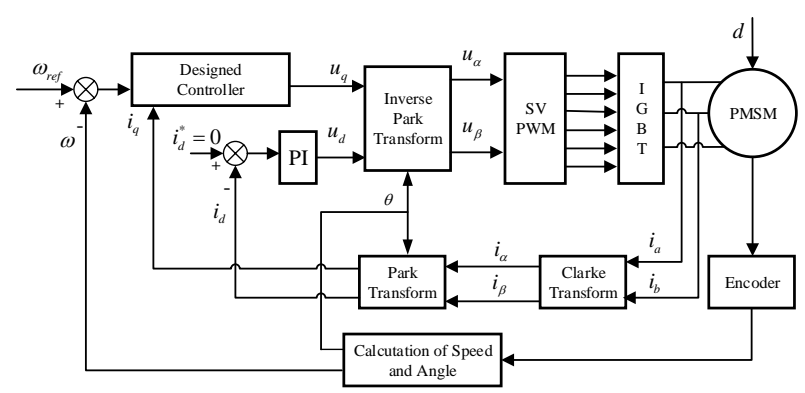

(a)

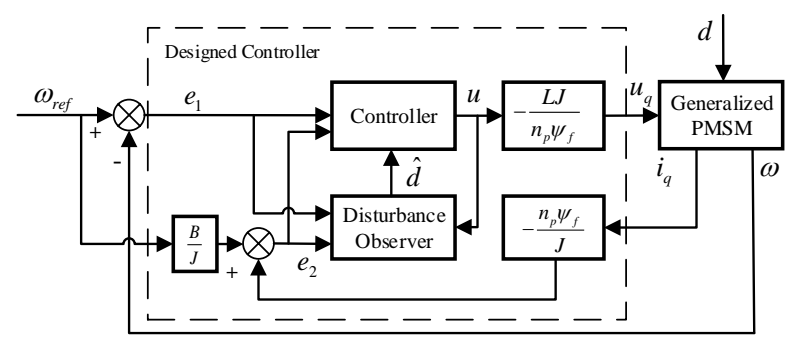

(b)

Fig. 2. The control diagram: (a) general drawing, (b) designed controller.

TABLE I

PARAMETERS OF THE PMSM System

\begin{tabular}{lll}
\hline \hline Symbol & Quantity & Nominal Values \\
\hline$n_{p}$ & Poles & 4 \\
$P_{N}$ & Rated power & $0.75 \mathrm{~kW}$ \\
$U_{N}$ & Rated voltage & $220 \mathrm{~V}$ \\
$I_{N}$ & Rated current & $4.7 \mathrm{~A}$ \\
$\omega_{N}$ & Rated rotating speed & $3000 \mathrm{rpm}$ \\
$T_{N}$ & Rated load torque & $2.387 \mathrm{~N} \cdot \mathrm{m}$ \\
$\psi_{f}$ & Flux linkage & $0.081 \mathrm{~Wb}$ \\
$J$ & Rotor inertia & $2.35 \times 10^{-4} \mathrm{~kg} \cdot \mathrm{m}^{2}$ \\
$B$ & Viscous damping & $7.4 \times 10^{-4} \mathrm{~N} \cdot \mathrm{m} \cdot \mathrm{s} / \mathrm{rad}$ \\
$R_{s}$ & Stator resistance & $0.8 \Omega$ \\
$L$ & Stator inductance & $2.9 \mathrm{mH}$ \\
\hline \hline
\end{tabular}

\section{Numerical Tests Results}

\section{A. Test Design}

In this section, numerical tests are performed using Matlab/Simulink. The specification is given in Table I. The rotating speed reference is $3000 \mathrm{rpm}$, the current constraint is $10 \mathrm{~A}$, and the voltage saturation limit is $220 \mathrm{~V}$.

In the simulation, the proposed method (16), i.e., the DOBCC controller, is compared with the conventional DOBC and the CCPID controller. The parameter tuning criterion should comprehensively take into account system stability, dynamic performance, current constraint satisfaction and robustness against unmatched disturbances. For fair comparisons, the control parameters of the DOB-CC, high-gain DOBC and CCPID controllers are tuned such that the speed tracking profiles have 
similar nominal performance specifications. While the lowgain DOBC is tuned to guarantee the current constraints in the whole process. The control parameters of the simulations are presented as follows: $k_{1}=8000, k_{2}=200, p=200$ for the high-gain DOBC; $k_{1}=6000, k_{2}=200, p=200$ for the low-gain DOBC; $k_{P}=8000, k_{D}=200, k_{I}=85000, l=50$ for the CCPID controller; and $k_{1}=8000, k_{2}=200, l=50$, $p=200$ for the DOB-CC controller;

\section{B. Numerical Results}

\section{- Test 1: at the start-up phase}

Fig. 3(a)-(c) are system responses of $e_{1}, i_{q}$ and $u_{q}$, respectively. To evaluate the control results qualitatively, the performance specification including overshoot (OS), settling time (ST) and peak current (PC) are utilized for validation. The performance indices are tabulated in Table II.

As shown in Fig. 3(a) and Table II, the speed response under the DOB-CC controller has comparable dynamic performance with the high-gain DOBC and CCPID controller, while the low-gain DOBC has longer ST. Regarding the current constraint problem, Fig. 3(b) and Table II illustrate that the DOB-CC and CCPID controllers keep a good balance between the speed regulation ability and satisfaction of the current constraint. While the PC of the high-gain DOBC exceeds constraint 10A. It indicates that the DOBC ensures the current constraint by selecting more conservative parameters. But this sacrifices the dynamic performance to some extent.

- Test 2: subject to different load torque disturbances

In what follows, we consider the current satisfaction and robustness under three controllers in the presence of the following three kinds of load torque disturbances.

1) Step form: The load torque $T_{L}$ increases from $1 \mathrm{~N} \cdot \mathrm{m}$ to $3 \mathrm{~N} \cdot \mathrm{m}$ at $t=0.4 \mathrm{~s}$ and decreases to $2.8 \mathrm{~N} \cdot \mathrm{m}$ at $t=0.7 \mathrm{~s}$.

2) Sawtooth form: The load torque $T_{L}$ repeatedly and monotonically increases from $2.8 \mathrm{~N} \cdot \mathrm{m}$ to $3.2 \mathrm{~N} \cdot \mathrm{m}$ and decreases to $2.8 \mathrm{~N} \cdot \mathrm{m}$, when $0.9 \leq t<1.5 \mathrm{~s}$.

3) Sinusoidal form: The load torque $T_{L}$ is imposed as $T_{L}=$ $0.3 \sin (20 \pi t)+0.2 \sin (10 \pi t)+2.8 \mathrm{~N} \cdot \mathrm{m}$ when $1.5 \leq t \leq$ $2 \mathrm{~s}$.

The system responses are shown in Fig. 3. Corresponding speed regulation performance indices, including peak current (PC), recovery time (RT) and root-mean-square steady-state error (RMSE), are utilized and shown in Table II.

TABLE II

PERFORMANCE INDICES IN NUMERICAL TESTS

\begin{tabular}{llllll}
\hline \hline Performance & OS(rpm) & ST(s) & PC(A) & RT(s) & RMSE(rpm) \\
\hline DOBC(high) & 0.206 & 0.072 & 10.268 & 0.048 & 34.904 \\
DOBC(low) & 0.202 & 0.116 & 9.956 & 0.090 & 40.772 \\
CCPID & 0.204 & 0.083 & 9.951 & 0.112 & 78.692 \\
DOB-CC & 0.203 & 0.079 & 9.953 & 0.055 & 35.606 \\
\hline \hline
\end{tabular}

As shown in Fig. 3(a) and Table II, the speed responses of the proposed DOB-CC controller and the high-gain DOBC are similar. While the low-gain DOBC and the CCPID controller have longer RT and generate larger RMSE. Fig. 3(b)

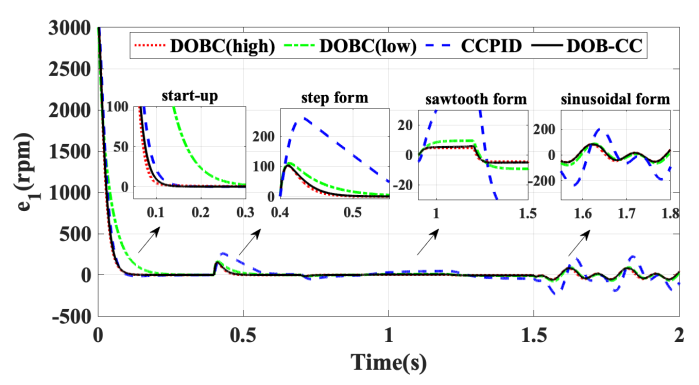

(a)

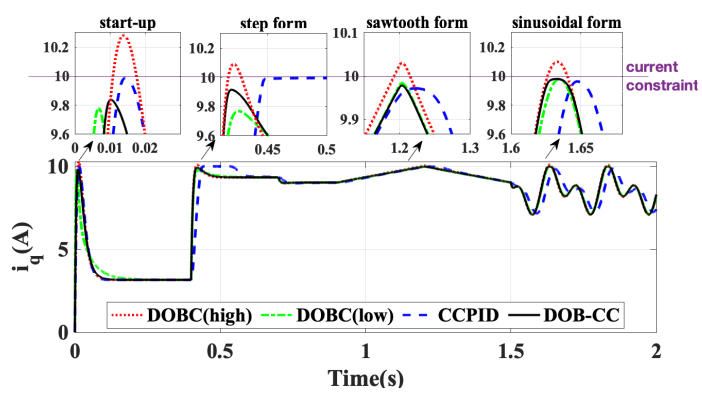

(b)

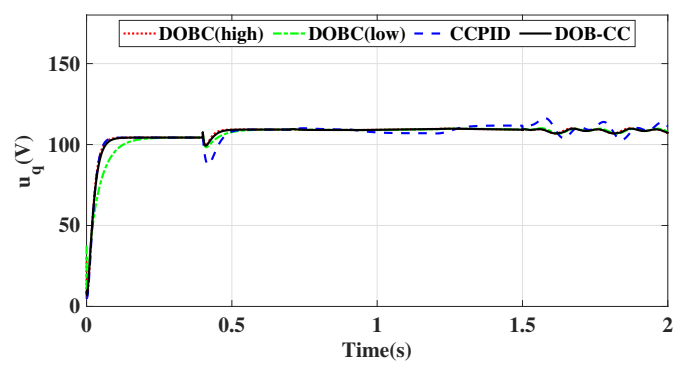

(c)

Fig. 3. System responses: (a) speed tracking error $e_{1}$, (b) q-axis current $i_{q}$, (c) q-axis voltage $u_{q} \cdot$ (simulations)

and Table II reveal that the DOB-CC controller ensures the current constraint 10A, while the high-gain DOBC violates the constraint. Moreover, in Fig. 3(c), the control efforts of the four controllers are kept at the same level for fair comparisons.

As shown in Tests 1 and 2, the proposed DOB-CC controller attains the best performance among three methods. It balances well between speed regulation performance and robustness, while satisfying the current constraint in the whole process.

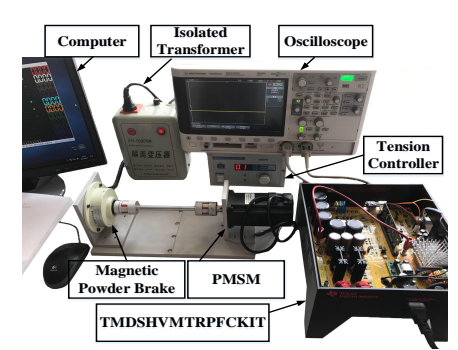

(a)

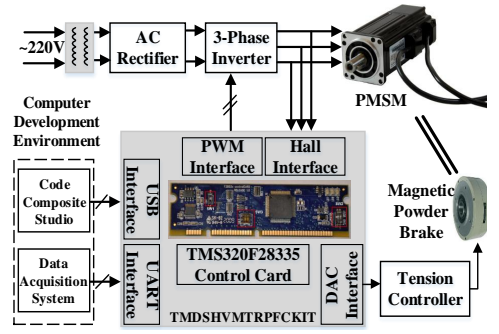

(b)
Fig. 4. Experiment system: (a) setup, (b) configuration. 


\section{EXPERIMENTAL TESTS RESUltS}

\section{A. Test Design}

For further validation, the experimental setup has been built and its configuration is shown in Fig. 4. The control and drive circuits are integrated in the platform TMDSHVMTRPFCKIT explored by Texas Instrument [40]. The current constraint is $10 \mathrm{~A}$, the voltage saturation limit is $220 \mathrm{~V}$, and the sampling frequency is $10 \mathrm{kHz}$. The control parameters are given as: $k_{1}=$ $7500, k_{2}=180, p=200$ for the high-gain DOBC; $k_{1}=5500$, $k_{2}=180, p=200$ for the low-gain DOBC; $k_{P}=7500$, $k_{D}=180, k_{I}=80000, l=50$ for the CCPID controller; and $k_{1}=7500, k_{2}=180, l=50, p=200$ for the DOB-CC controller.

\section{B. Experimental Results}

- Test 1: at the start-up phase

Figs. 5(a)-(c) are system responses of $e_{1}, i_{q}$ and $u_{q}$, respectively. The corresponding performance indices are presented in Table III. Consistent with the numerical results, it is observed in Fig. 5 and Table III that the proposed DOB-CC controller achieves comparable dynamic performance with CCPID controller, i.e., similar OS and ST. Both PCs of them are lower than 10A, which indicates that the proposed DOB-CC and CCPID controllers ensure the current constraint effectively. While the DOBC cannot guarantee the current constraint under the same dynamic performance requirement.

\section{- Test 2: subject to different load torque disturbances}

Corresponding with the numerical tests, the following load torque disturbances are taken into account in the experiments.

1) Step form: The load torque $T_{L}$ increases from $1 \mathrm{~N} \cdot \mathrm{m}$ to $3 \mathrm{~N} \cdot \mathrm{m}$ at $t=2.2 \mathrm{~s}$ and decreases to $2.8 \mathrm{~N} \cdot \mathrm{m}$ at $t=2.6 \mathrm{~s}$.

2) Sawtooth form: The load torque $T_{L}$ monotonically increases from $2.8 \mathrm{~N} \cdot \mathrm{m}$ to $3.2 \mathrm{~N} \cdot \mathrm{m}$ and decreases to $2.8 \mathrm{~N} \cdot \mathrm{m}$ when $3.2 \leq t \leq 3.8 \mathrm{~s}$.

3) Sinusoidal form: The load torque $T_{L}=0.3 \sin (20 \pi t)+$ $0.2 \sin (10 \pi t)+2.8 \mathrm{~N} \cdot \mathrm{m}$ is imposed when $4 \leq t \leq 5 \mathrm{~s}$.

The system responses are shown in Figs. 6-8, and Table III presents specific speed regulation performance indices.

TABLE III

PERFORMANCE INDICES IN EXPERIMENTAL TESTS

\begin{tabular}{llllll}
\hline \hline Performance & OS(rpm) & ST(s) & PC(A) & RT(s) & RMSE(rpm) \\
\hline DOBC(high) & 2.985 & 0.117 & 11.309 & 0.113 & 35.758 \\
DOBC(low) & 2.263 & 0.160 & 9.612 & 0.137 & 41.363 \\
CCPID & 2.932 & 0.123 & 9.609 & 0.242 & 80.453 \\
DOB-CC & 2.763 & 0.120 & 9.615 & 0.116 & 36.161 \\
\hline \hline
\end{tabular}

As shown in Figs. 6(a), 7(a) and 8(a) and Table III, the RT and RMSE of the DOB-CC controller and the high-gain DOBC are comparable, and smaller than those of the lowgain DOBC and CCPID controller. Table III and Figs. 6(b), 7(b) and 8(b) illustrate that the DOB-CC controller ensures the current constraint 10A, while the high-gain DOBC fails to do so. Furthermore, Figs. 6(c), 7(c) and 8(c) indicate that each control effort is kept at the same level.
Hence, the experimental results further verify the effectiveness of the proposed DOB-CC method, which can simultaneously realize the current constraint task and compensate the undesirable effects of unmatched load torque variations, while maintaining satisfying speed tracking performance.

\section{CONCLUSIONS}

The overcurrent protection and unmatched disturbance rejection problems have been investigated simultaneously for speed regulation of PMSM systems. A DOB-CC controller has been developed via a DOB and a constraint coping mechanism. Comprehensive theoretical analysis, together with numerical and experimental validation, have demonstrated its effectiveness and feasibility. A promising future research direction is to extend the proposed method to a higher-order system with varying state constraints and control input saturations subject to matched/unmatched disturbances.

\section{REFERENCES}

[1] D. Seo, Y. Bak, and K.-B. Lee, "An improved rotating restart method for a sensorless permanent-magnet synchronous motor drive system using repetitive zero voltage vectors," IEEE Trans. Ind. Electron., DOI 0.1109/TIE.2019.2914647, 2019.

[2] V. M. Hernández-Guzmán and J. Orrante-Sakanassi, "PID control of robot manipulators actuated by BLDC motors," Int. J. Control, DOI 10.1080/00207179.2019.1590648, pp. 1-10, 2019.

[3] X. Zhao and S. Niu, "A new slot-PM vernier reluctance machine with enhanced zero sequence current excitation for electric vehicle propulsion," IEEE Trans. Ind. Electron., DOI 10.1109/TIE.2019.2920600, 2019.

[4] Q. Tan, X. Huang, L. Li, and M. Wang, "Magnetic field analysis and flux barrier design for modular permanent magnet linear synchronous motor," IEEE Trans. Ind. Electron., DOI 10.1109/TIE.2019.2918477, 2019.

[5] S. Bolognani, S. Bolognani, L. Peretti, and M. Zigliotto, "Design and implementation of model predictive control for electrical motor drives," IEEE Trans. Ind. Electron., vol. 56, no. 6, pp. 1925-1936, 2009.

[6] T. Tarczewski and L. M. Grzesiak, "Constrained state feedback speed control of PMSM based on model predictive approach," IEEE Trans. Ind. Electron., vol. 63, no. 6, pp. 3867-3875, 2016.

[7] Z. Hu and K. Hameyer, "A method of constraint handling for speedcontrolled induction machines," IEEE Trans. Ind. Electron., vol. 63, no. 7, pp. 4061-4072, 2016.

[8] M. Brasel, "A gain-scheduled multivariable LQR controller for permanent magnet synchronous motor," in Proc. IEEE MMAR Conf., pp. 722 $725,2014$.

[9] M. Preindl and S. Bolognani, "Model predictive direct speed control with finite control set of PMSM drive systems," IEEE Trans. Power Electron., vol. 28, no. 2, pp. 1007-1015, 2013.

[10] R. Krishnan, Electric motor drives: modeling, analysis and control. Prentice Hall, 2001.

[11] Z. Sun, S. Li, J. Wang, X. Zhang, and X. Mo, "Adaptive composite control method of permanent magnet synchronous motor systems," Trans. Inst. Meas. Control, vol. 40, no. 11, pp. 3345-3357, 2018.

[12] A. Rauf, J. Yang, R. Madonski, S. Li, and Z. Wang, "Sliding mode control of converter-fed DC motor with mismatched load torque compensation," in Proc. 28th IEEE Int. Symp. Ind. Electron., pp. 653-657. IEEE, 2019

[13] J. Yang, H. Wu, L. Hu, and S. Li, "Robust predictive speed regulation of converter-driven DC motors via a discrete-time reduced-order GPIO," IEEE Trans. Ind. Electron., vol. 66, no. 10, pp. 7893-7903, 2018.

[14] R. L. Kosut, "Design of linear systems with saturating linear control and bounded states," IEEE Trans. Autom. Control, vol. 28, no. 1, pp. 121-124, 1983.

[15] H. Fang and Z. Lin, "Stability analysis for linear systems under state constraints," IEEE Trans. Autom. Control, vol. 49, no. 6, pp. 950-955, 2004.

[16] M. Vassilaki, J. Hennet, and G. Bitsoris, "Feedback control of linear discrete-time systems under state and control constraints," Int. J. Control, vol. 47, no. 6 , pp. $1727-1735,1988$. 


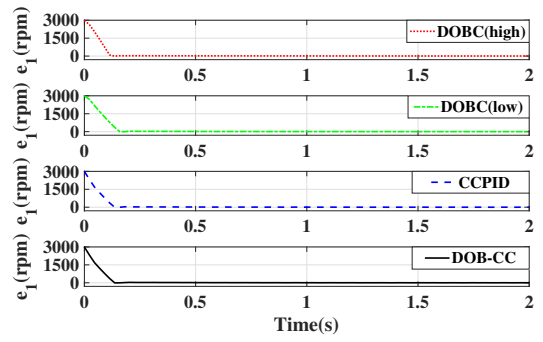

(a)

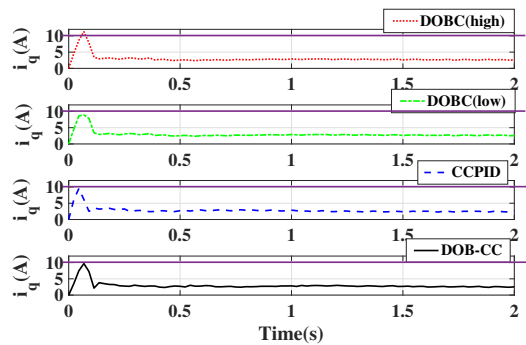

(b)

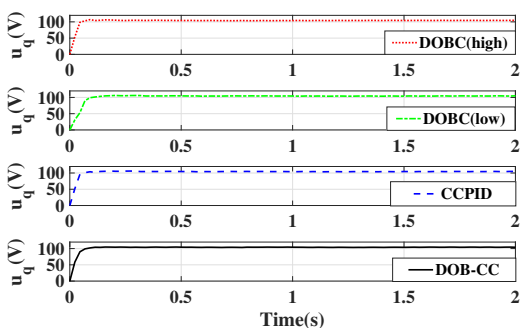

(c)

Fig. 5. System responses at the start-up phase: (a) speed tracking error $e_{1}$, (b) q-axis current $i_{q}$, (c) q-axis voltage $u_{q}$. (experiments)

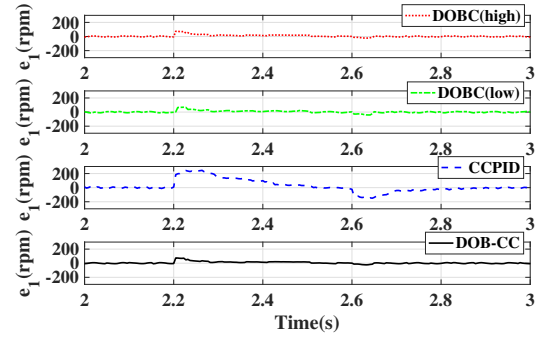

(a)

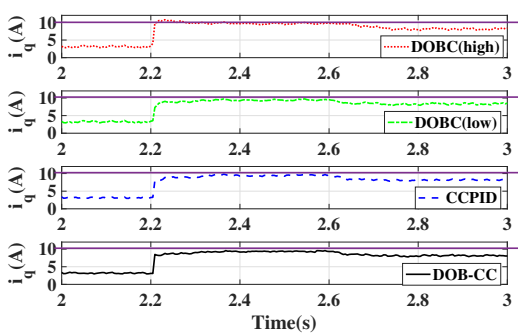

(b)

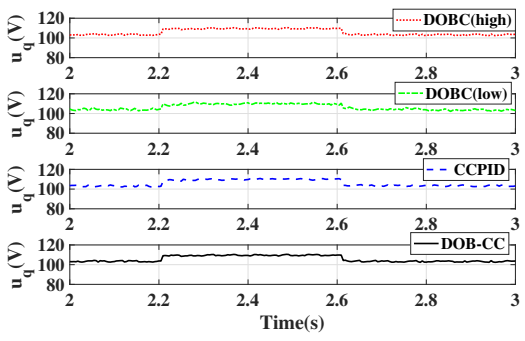

(c)

Fig. 6. System responses with a step load toque: (a) speed tracking error $e_{1}$, (b) q-axis current $i_{q}$, (c) q-axis voltage $u_{q}$. (experiments)

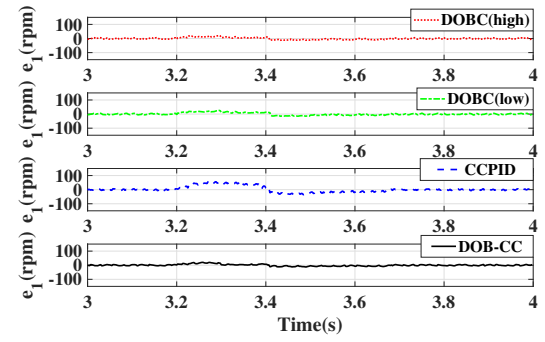

(a)

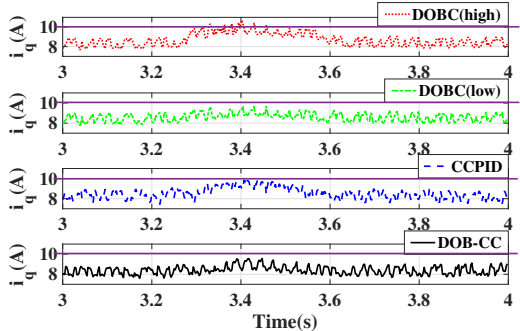

(b)

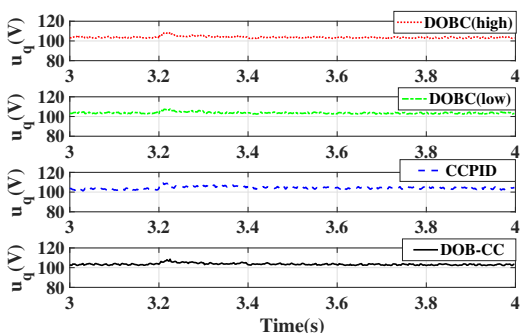

(c)

Fig. 7. System responses with a sawtooth load toque: (a) speed tracking error $e_{1}$, (b) q-axis current $i_{q}$, (c) q-axis voltage $u_{q}$. (experiments)

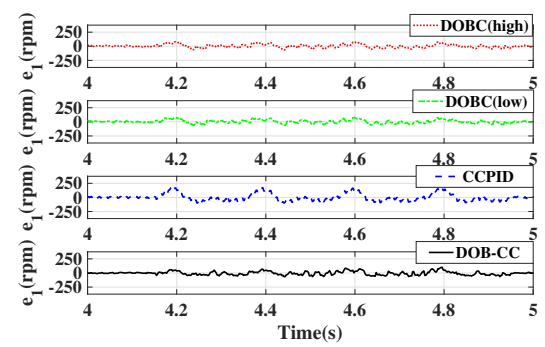

(a)

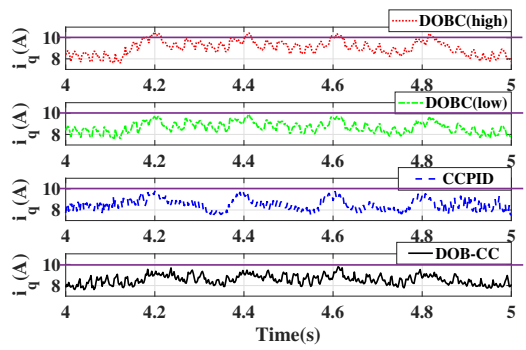

(b)

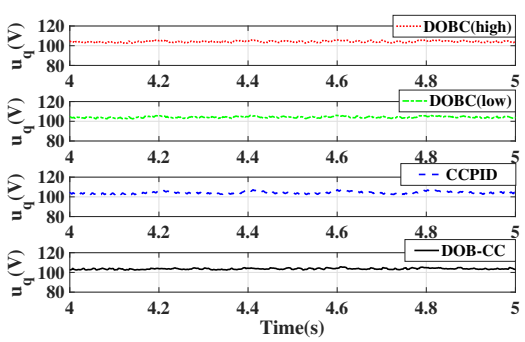

(c)

Fig. 8. System responses with a sinusoidal load torque: (a) speed tracking error $e_{1}$, (b) q-axis current $i_{q}$, (c) q-axis voltage $u_{q}$. (experiments) 
[17] S. Li, Y. Yan, J. Yang, Z. Sun, and H. Yu, "Nonlinear-disturbanceobserver-enhanced MPC for motion control systems with multiple disturbances," IET Control Theory Appl., 2019.

[18] A. G. Wills and W. P. Heath, "Barrier function based model predictive control," Automatica, vol. 40, no. 8, pp. 1415-1422, 2004.

[19] J. Yang, W. X. Zheng, S. Li, B. Wu, and M. Cheng, "Design of a prediction-accuracy-enhanced continuous-time MPC for disturbed systems via a disturbance observer," IEEE Trans. Ind. Electron., vol. 62, no. 9, pp. 5807-5816, 2015.

[20] P. Mhaskar, N. H. El-Farra, and P. D. Christofides, "Stabilization of nonlinear systems with state and control constraints using Lyapunovbased predictive control," Syst. Control Lett., vol. 55, no. 8, pp. 650-659, 2006.

[21] L. Chen and Q. Wang, "Prescribed performance-barrier Lyapunov function for the adaptive control of unknown pure-feedback systems with full-state constraints," Nonlinear Dynamics, vol. 95, no. 3, pp. 2443 2459, 2019.

[22] W. Sun, S.-F. Su, Y. Wu, J. Xia, and V.-T. Nguyen, "Adaptive fuzzy control with high-order barrier Lyapunov functions for high-order uncertain nonlinear systems with full-state constraints," IEEE Trans. Cybern., DOI 10.1109/TCYB.2018.2890256, 2019.

[23] Y.-J. Liu and S. Tong, "Barrier Lyapunov functions-based adaptive control for a class of nonlinear pure-feedback systems with full state constraints," Automatica, vol. 64, pp. 70-75, 2016.

[24] K. P. Tee, S. S. Ge, and F. E. H. Tay, "Adaptive control of electrostatic microactuators with bidirectional drive," IEEE Trans. Control Syst. Technol., vol. 17, no. 2, pp. 340-352, 2009.

[25] D. Won, W. Kim, D. Shin, and C. C. Chung, "High-gain disturbance observer-based backstepping control with output tracking error constraint for electro-hydraulic systems," IEEE Trans. Control Syst. Technol., vol. 23, no. 2, pp. 787-795, 2015.

[26] W. He, S. Zhang, and S. S. Ge, "Adaptive control of a flexible crane system with the boundary output constraint," IEEE Trans. Ind. Electron., vol. 61, no. 8, pp. 4126-4133, 2014.

[27] T. Guo, Z. Wang, X. Wang, S. Li, and Q. Li, "A simple control approach for buck converters with current-constrained technique," IEEE Trans. Control Syst. Technol., vol. 27, no. 1, pp. 418-425, 2017.

[28] T. Guo, Z. Sun, X. Wang, S. Li, and K. Zhang, "A simple currentconstrained controller for permanent-magnet synchronous motor," IEEE Trans. Ind. Informat., vol. 15, no. 3, pp. 1486-1495, 2019.

[29] K. Ohishi, M. Nakao, K. Ohnishi, and K. Miyachi, "Microprocessorcontrolled DC motor for load-insensitive position servo system," IEEE Trans. Ind. Electron., vol. 34, no. 1, pp. 44-49, 1987.

[30] J. Yang, T. Li, C. Liu, S. Li, and W.-H. Chen, "Nonlinearity estimatorbased control of a class of uncertain nonlinear systems," IEEE Trans. Autom. Control, DOI 10.1109/TAC.2019.2940567, 2019.

[31] J. Yang, W.-H. Chen, S. Li, L. Guo, and Y. Yan, "Disturbance/uncertainty estimation and attenuation techniques in PMSM drives-A survey," IEEE Trans. Ind. Electron., vol. 64, no. 4, pp. 3273-3285, 2017.

[32] Z. Tian, Q.-C. Zhong, B. Ren, and J. Yuan, "UDE-based robust control for systems with mismatched uncertainties via feedback compensation," Int. J. Control, DOI 10.1080/00207179.2019.1669826, pp. 1-11, 2019.

[33] L. Guo and X.-Y. Wen, "Hierarchical anti-disturbance adaptive contro for non-linear systems with composite disturbances and applications to missile systems," IEEE Trans. Inst. Meas. Control, vol. 33, no. 8, pp. 942-956, 2011.

[34] X.-J. Wei, Z.-J. Wu, and H. R. Karimi, "Disturbance observer-based disturbance attenuation control for a class of stochastic systems," Automatica, vol. 63, pp. 21-25, 2016.

[35] H. Zhang, X. Wei, L. Zhang, and M. Tang, "Disturbance rejection for nonlinear systems with mismatched disturbances based on disturbance observer,' J. Franklin Inst., vol. 354, no. 11, pp. 4404-4424, 2017.

[36] T. Guo, S. Huang, and X. Wang, "Overcurrent protection control design for DC-DC buck converter with disturbances," IEEE Access, vol. 7, pp. 90 825-90 833, 2019

[37] Z. Sun, T. Guo, Y. Yan, X. Wang, and S. Li, "A composite currentconstrained control for permanent magnet synchronous motor with timevarying disturbance," Adv. Mech. Eng., vol. 9, no. 9, pp. 1-13, 2017.

[39] S. Bolognani, R. Oboe, and M. Zigliotto, "Sensorless full-digital PMSM drive with EKF estimation of speed and rotor position," IEEE Trans. Ind. Electron., vol. 46, no. 1, pp. 184-191, 1999.
[38] Z. Sun, Y. Zhang, S. Li, and X. Zhang, "A simplified composite currentconstrained control for permanent magnet synchronous motor speedregulation system with time-varying disturbances," Trans. Inst. Meas. Control, vol. 42, no. 3, pp. 374-385, 2020.

[40] J. Mao, J. Yang, S. Li, Y. Yan, and Q. Li, "Output feedback-based sliding mode control for disturbed motion control systems via a higherorder ESO approach," IET Control Theory Appl., vol. 12, no. 15, pp. 2118-2126, 2018

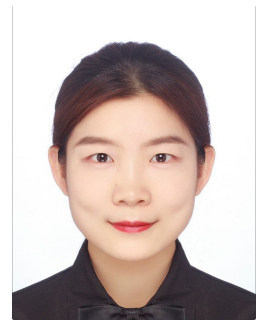

Chen Dai (M'19) received her B.S. and M.S. degrees both in Automatic Control from Southeast university, Nanjing, China in 2016 and 2018, respectively. She is currently a Ph.D student of Mechatronic Systems Control Laboratory of School of Automation, Southeast University. Her main research interests include disturbance estimation and compensation, constrained-system control and nonlinear control theory with applications to mechatronic systems.

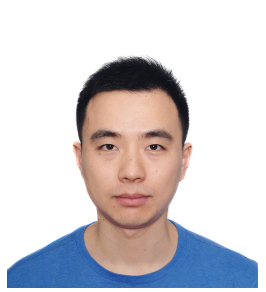

Tianliang Guo received his B.S. degree in Mathematics from Hunan University of Science and Technology in 2010, and his M.S. degree in Mathematics from Anhui University in 2013. In 2019, he received his Ph.D. degree in control theory and control engineering from School of Automation, Southeast University, Nanjing, China. His current research interests include constrained-system control and applications, output feedback control and interconnected systems.

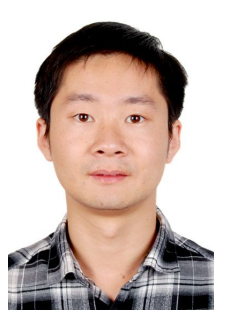

Jun Yang (SM'18) received his B.S. degree in the Department of Automatic Control from Northeastern University, Shenyang, China in 2006. In 2011, he received his Ph.D. degree in control theory and control engineering from School of Automation, Southeast University, Nanjing, China, where he is currently a Professor. His research interests include disturbance estimation and compensation, advanced control theory and its application to flight control systems and motion control systems.

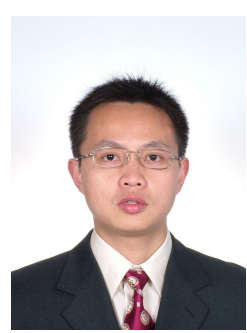

Shihua Li (SM'10-F'19) received his B.S., M.S. and Ph.D. degrees all in Automatic Control from Southeast university, Nanjing, China in 1995 1998 and 2001, respectively. Since 2001, he has been with School of Automation, Southeast University, where he is currently a professor and the director of Mechatronic Systems Contro Laboratory. His main research interests lie in modeling, analysis and nonlinear control theory with applications to mechatronic systems, including manipulator, robot, AC motor, power electronic systems and others. 